This item was submitted to Loughborough's Research Repository by the author.

Items in Figshare are protected by copyright, with all rights reserved, unless otherwise indicated.

\title{
Shared control for the kinematic and dynamic models of a mobile robot
}

\section{PLEASE CITE THE PUBLISHED VERSION}

https://doi.org/10.1109/tcst.2016.2528890

\section{PUBLISHER}

(C) Institute of Electrical and Electronics Engineers (IEEE)

\section{VERSION}

AM (Accepted Manuscript)

\section{LICENCE}

CC BY-NC-ND 4.0

\section{REPOSITORY RECORD}

Jiang, Jingjing, Pierluigi Di Franco, and Alessandro Astolfi. 2019. "Shared Control for the Kinematic and Dynamic Models of a Mobile Robot”. figshare. https://hdl.handle.net/2134/36752. 


\title{
Shared-Control for the Kinematic and the Dynamic Model of a Mobile Robot
}

\author{
Jingjing Jiang*, Pierluigi Di Franco \\ and Alessandro Astolfi, Fellow, IEEE
}

\begin{abstract}
This paper presents shared-control algorithms for the kinematic and the dynamic models of a mobile robot with a feasible configuration set defined by means of linear inequalities. The shared-control laws based on a hysteresis switch are designed in the case in which absolute positions are not available. Instead, we measure the distances to obstacles and angular differences. Formal properties of the closed-loop systems with the sharedcontrol are established by a Lyapunov-like analysis. Simulation results and experimental results are presented to show the effectiveness of the algorithm.
\end{abstract}

\section{INTRODUCTION}

Mobile robots are machines with the ability of locomotion [1]. They are widely used in industry, search and rescue, military and domestic fields. For instance, mobile robots are used in many warehouses to transfer materials from shelves to loading/unloading areas and vice-versa. Modern troops are equipped with mobile robots for reconnaissance missions and to attack the enemy. We are also able to find domestic robots that perform certain housekeeping tasks, such as vacuuming, window washing and gardening, being sold on the market. Furthermore, wheelchairs and other assistive devices can also be regarded as mobile robots.

Our study is motivated by the design of smart wheelchairs that are used to help people with mobility disabilities to move safely in their daily life [2]. The shared-controller that combines the user input and the feedback control input is installed to reduce the number of accidents and to correct the user's "perilous" behaviours. Note that the name "sharedcontrol" has the same meaning as that in [3] and as in the anti-lock braking system. The wheelchair is mostly "driven" by the operator except for "dangerous" situations, in which the feedback controller takes charge of the system. It aims to integrate the best of both worlds: the reliable performance of a feedback controller and the adaptive, interactive and inventive task execution ability of a human operator. Therefore it is very helpful to the disabled.

Numerous studies and experiments have been performed on

*J. Jiang (the corresponding author) is with the Department of Electrical and Electronic Engineering, Imperial College London, UK, E-mail: jingjing.jiang10@imperial.ac.uk

P. Di Franco is with the DICII, University of Roma "Tor Vergata", Via del Politecnico 1, 00133 Rome, Italy, E-mail: pierluigi.di-francol3eimperial.ac.uk

A. Astolfi is with the Department of Electrical and Electronic Engineering, Imperial College London, London, SW7 2AZ, UK and the DICII, University of Roma "Tor Vergata", Via del Politecnico 1, 00133 Rome, Italy, E-mail: a.astolfi@imperial.ac.uk mobile robots: we only list here a few of them. ProportionalIntegral-Derivative (PID) controllers are widely used in the industry as well as in the control of mobile robots. The paper [4] has introduced a laser guidance control based on a PD controller to inspect the welds in a nuclear power plant. To avoid collisions, the potential field method is used as discussed in [5], where an attractive force and a repulsive force applied to the robot are generated by the target position and the obstacles, respectively. This may not work well in circumstance in which the combination of a global path generation and a local reactive trajectory generation, based on a focused $D^{*}$ search and an obstacle avoider, respectively, can be used to control the robot [6]. Even though the robot is able to avoid hitting obstacles, it is unable to reach them. Model Predictive Control (MPC) is another popular method utilized in the design of tracking controller for mobile robots as explained in [7] and [8]. These MPC designs rely on full information on the environment to build the artificial potential and may need large computation for complex environments. $H_{\infty}$ control and sliding mode control have also been implemented in the control of mobile robots. For instance, [9] has used nonlinear $H_{\infty}$ control via quasi-linear parameter varying representation to control wheeled robots and [10] has presented an implementation of integral sliding mode controller on a two-wheeled mobile robot. Other control methods, such as back-stepping control [11], adaptive control [12], [13], fuzzy control [14], [15] and control based on the representation as chained system [16], [17], [18], have also been explored and implemented.

The references introduced in the previous paragraph are on how to design a feedback controller for the automatic navigation of a mobile robot. However, if the human operator is included in the closed-loop system, the situation becomes more complicated. To combine the human input and the feedback control input together, a shared-control algorithm based on the hysteresis switch has been introduced in [19]. The paper [20] has proposed steering-like and brake-like functions according to the distance to the obstacles to avoid collisions, while the human operator drives the robot with a joystick and the stability of the system is analyzed through a linear model. The shared-control problem for a mobile robot has been studied in [21], where the shared controller is used to cope with lowlevel navigations and thus reduces the operator's workload. However, the human's competence is not improved. HumanRobot bilateral shared-control has been used in [22], where the robot is able to move autonomously according to the operator's order and a haptic force feedback is available to the human operator indicating the deformation on the desired path. One way to generate the haptic force has been introduced in [23]. 
Note that high requirements on the human operator is essential for commanding direct motion inputs in a cluttered environment. To reduce the burden of directly driving the robot, the paper [24] has proposed a semi-autonomous algorithm at the planning level. Furthermore, a reactive planning approach to bilaterally teleoperate a UAV has been presented in [25]. Therein the human operator is allowed to modify the path and an autonomous feedback controller is used to assist the human in reaching the target position. A semi-autonomous UAV platform has been presented in [26], in which the hapic feedback reflecting the environment and the system states is available to the human operator. A shared-control law for the kinematic model of a mobile robot with knowledge of the absolute positions is given in [27]. This paper extends the results of [27] to the cases in which absolute positions are not measurable and the shared-control algorithm has to deal with both static and (non-predefined) dynamic environments. It provides formal proofs of all technical statements, includes additional theoretical results and case studies. Both the simulation results and the experimental ones demonstrate that the robot with the shared-controller moves "safely" without hitting any obstacle. If the feedback controller shares the same objective as the operator, it is used to help the robot tracking the trajectory safely (Cases I and II in Section V). Otherwise, the feedback controller would regard the predicted human behaviour as its goal (Case III in Section V).

The rest of the paper is organized as follows. The sharedcontrol problem is formulated in Section II, which also provides a few definitions and assumptions. Novel algorithms to solve the shared-control problem for the kinematic model and the dynamic model of the mobile robot are given in Section III and IV, respectively. Formal properties of the closed-loop systems with the shared-control algorithm are also established. Section V presents three case studies to illustrate the performance of the shared-control law through simulation and experiments. Finally, conclusions and suggestions for future work are given in Section VI.

\section{PROBLEM FORMULATION, DEFINITIONS AND ASSUMPTIONS}

This section formulates the shared-control problem for the kinematic and the dynamic models of a unicycle robot. The kinematic model, a simple and common way to study the motion of a wheeled robot, is widely used in the navigation in combination with some low-level controls. It is also the basis of the dynamic model, the control of which is more difficult. However, robots are driven by motors which produce torques, directly relative to the acceleration rather than the velocity of the robots. The dynamic model may be used to describe the situations in which the kinematic model has to complemented with some additional dynamics, for example those due to the presence of actuators. Therefore, this paper studies both the kinematic and the dynamic models and provides sharedcontrol algorithms for these models.

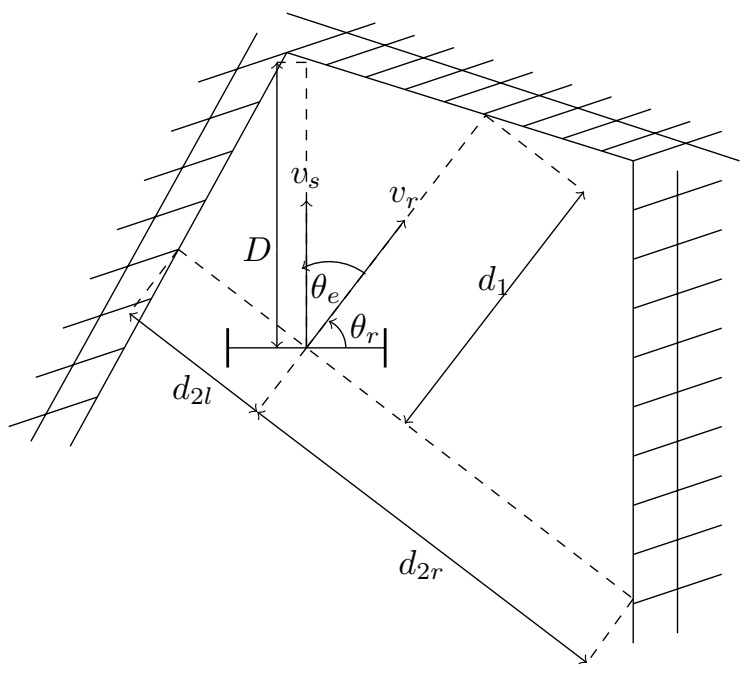

Fig. 1. Definitions of $d_{1}, d_{2 l}, d_{2 r}$ and $\theta_{e}$ (shadowed region: unfeasible region, $v_{r}$ : reference forward velocity).

The kinematic and the dynamic models of a wheeled mobile robot can be described by the equations

$$
\begin{aligned}
& \dot{x}=v_{s} \cos \theta, \\
& \dot{y}=v_{s} \sin \theta, \\
& \dot{\theta}=\omega_{s},
\end{aligned}
$$

and

$$
\begin{aligned}
\dot{x} & =v \cos \theta, \\
\dot{y} & =v \sin \theta, \\
\dot{\theta} & =\omega, \\
\dot{v} & =u_{1 s} / m, \\
\dot{\omega} & =u_{2 s} / I,
\end{aligned}
$$

respectively, where $(x, y)$ denotes the Cartesian coordinates of the center of mass of the robot, $\theta$ denotes the angle between the (positive) heading direction and the $x$-axis, $m$ and $I$ represent the mass and the moment of the inertia of the robot, respectively. $v_{s}$, the linear forward velocity, and $\omega_{s}$, the angular velocity, are external inputs for the system (1), while $v$ and $\omega$ are states for the system (2), although still describing the linear forward velocity and the angular velocity, respectively. In addition, $u_{1 s}$ and $u_{2 s}$ represent the generalized force variable and the steering torque for the system (2).

In [27] a shared-control algorithm for a mobile robot with the knowledge of absolute positions has been given. This paper focuses on the shared-control problem without measurement of absolute positions. Instead, the measurements are distances to obstacles along (i.e. $d_{1}$ ) and orthogonal to (i.e. $d_{2}$ defined at the end of this paragraph) the direction of $v_{r}$ and the difference between the real and the reference heading angle (i.e. $\theta_{e}=$ $\theta-\theta_{r}$ ) (see Figure 1). Note that $v_{r}$ is the reference linear velocity of the robot. The definition of $d_{2}$ is given as

$$
d_{2}= \begin{cases}d_{2 l} & \text { if }\left|\log \frac{d_{2 l}}{d_{r_{2 l}}}\right| \leq\left|\log \frac{d_{2 r}}{d_{r_{2 r}}}\right|, \\ d_{2 r} & \text { if }\left|\log \frac{d_{2 l}}{d_{r_{2 l}}}\right|>\left|\log \frac{d_{2 r}}{d_{r_{2 r}}}\right|,\end{cases}
$$




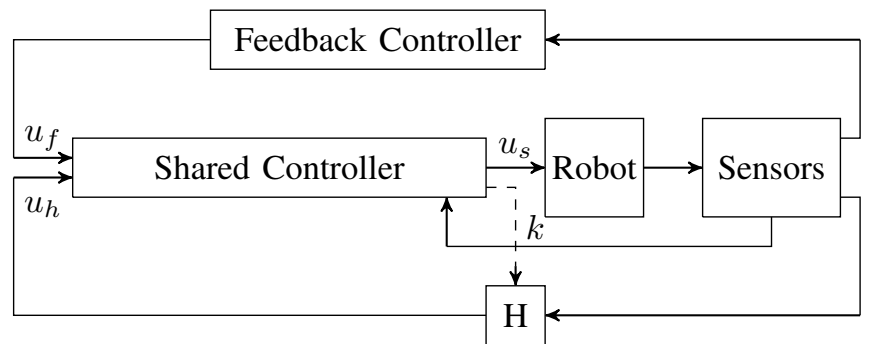

Fig. 2. The proposed control architecture for the closed-loop system with shared control.

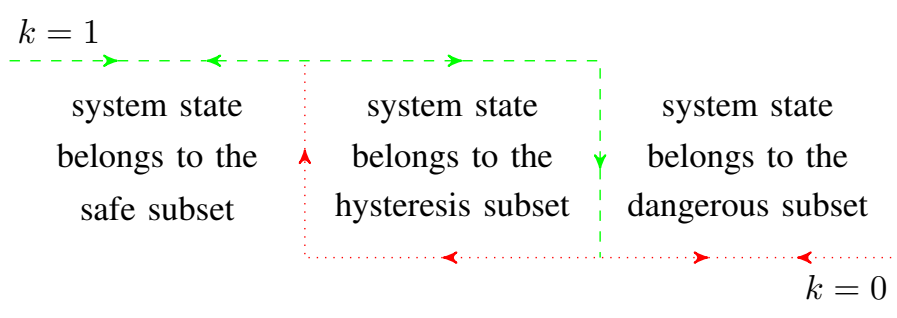

Fig. 3. The sharing function $k: k=1$ (green, dashed) and $k=0$ (red, dotted).

where $d_{r_{2 l}}$ and $d_{r_{2 r}}$ are defined in what follows in equation (8).

In the rest of this paper we use the subscripts $h, f$ and $s$ to denote the control inputs generated by the human operator, the feedback controller and the shared-controller, respectively.

Definition 1: We use the name $h$-control and f-control, denoted as $u_{h}$ and $u_{f}$, to describe the human action and the feedback control action, respectively. In addition, the sharing function, denoted as $k$, is used to quantify how the control authority is shared between $u_{h}$ and $u_{f}$.

The control architecture is given in Fig. 2, where 'H' describes the human action. Note that the function $k \in[0,1]$ can be used as a feedback signal to the human operator indicating how dangerous the situation is: this is helpful to the human operator in improving his/her behaviours. One possible selection for $k$ is a hysteresis switch, as illustrated in Fig. 3. The overall set describing the feasible values of the state can be divided into three subsets, the safe subset, the hysteresis subset and the dangerous subset. The variable $k=1$ if the state belongs to the safe subset or if the state enters the hysteresis subset from the safe subset; $k=0$ if the state belongs to the dangerous subset or if the state enters the hysteresis subset from the dangerous subset. In addition, as illustrated in Fig. 2 the external input of the mobile robot is the shared control signal $u_{s}$ which is a combination of the feedback control input $u_{f}$ and the human input $u_{h}$.

Definition 2: The s-closed-loop and the h-closed-loop for the kinematic model of a mobile robot are used to denote the system described by (1) and

$$
\begin{aligned}
\dot{x} & =v_{h} \cos \theta, \\
\dot{y} & =v_{h} \sin \theta, \\
\dot{\theta} & =\omega_{h},
\end{aligned}
$$

respectively, where $v_{h}$ and $\omega_{h}$ are the inputs generated by the human operator. Similarly, the s-closed-loop and the h-closedloop for the dynamic model of a mobile robot are used to denote the system described by (2) and

$$
\begin{aligned}
& \dot{x}=v \cos \theta, \\
& \dot{y}=v \sin \theta, \\
& \dot{\theta}=\omega, \\
& \dot{v}=u_{1 h} / m, \\
& \dot{\omega}=u_{2 h} / I,
\end{aligned}
$$

respectively, where $u_{1 h}$ and $u_{2 h}$ are the human inputs. In addition, $\Omega_{h}$ and $\Omega_{s}$ denote the $\Omega$-limit set of the h-closedloop and s-closed-loop, respectively.

Definition 3: The function $f(y(t), x(t), t)=\operatorname{atg}(y(t), x(t), t)$ is a continuous function defined as

$$
\operatorname{atg}(y(t), x(t), t)=\operatorname{atan}(y(t), x(t))+2 \alpha(t) \pi,
$$

where atan is the four quadrant arctan function, $\alpha(0)=0$ and

$$
\alpha(t)= \begin{cases}\alpha(t-\delta t)+1, & \text { if } a=-2 \pi \\ \alpha(t-\delta t)-1, & \text { if } a=2 \pi \\ \alpha(t-\delta t), & \text { else }\end{cases}
$$

with $a=\lim _{\delta t \rightarrow 0^{+}} \operatorname{atan}(y(t), x(t))-\operatorname{atan}(y(t-\delta t), x(t-\delta t))$ for all $t>0$.

Note that Definition 3 is similar to the definition of the standard four quadrant arctan function except that the function $\operatorname{atg}(\cdot)$ takes values in $(-\infty, \infty)$ rather than in $[-\pi, \pi)$. The following example can be used to help understanding Definition 3. Suppose the robot is tracking a unity circle centered at the origin with angular speed equals to 1, i.e. $x(t)=\cos (t), y(t)=\sin (t)$. Then for any $t \in[0, \pi], \operatorname{atan}(y(t), x(t))=t$ and $\alpha(t)=0$. However, $\lim _{\delta t \rightarrow 0^{+}} \operatorname{atan}(y(\pi+\delta t), x(\pi+\delta t))=-\pi$ indicating $\lim _{\delta t \rightarrow 0^{+}} \operatorname{atan}(y(\pi+\delta t), x(\pi+\delta t))-\operatorname{atan}(y(\pi), x(\pi))=-2 \pi$. Therefore, $\alpha(\pi+\delta t)=1$. It is therefore easy to conclude that, in the example, $\alpha(t)=i$ if $t \in(2 i-\pi, 2 i+\pi]$, with $i$ a positive integer.

Assumption 1: The admissible Cartesian configuration set for the robot, denoted as $\mathcal{P}_{a}$, is non-empty.

Assumption 2: The function $d_{d}(t)=\left[d_{d 1}(t), d_{d 2}(t)\right]^{T}$, representing the desired distances to the relative obstacles (the obstacles along and orthogonal to the direction of $v_{r}$ ), is continuous.

Assumption 3: To simplify the calculation, we assume that the mass $m$ and the moment of inertia $I$ of the mobile robot are equal to 1 .

The above assumptions hold for the rest of the paper. 
Suppose $\mathcal{P}_{a} \subset \mathcal{P}=\mathbb{R}^{2}$ is a given, closed and compact set describing the admissible Cartesian configurations for the system (1) and $u_{h}$ is a given h-control. The shared-control problem for the kinematic model of a mobile robot can be formulated as follows.

Given the system (1), an admissible configuration set $\mathcal{P}_{a}$ and an h-control, find (if possible)

- an f-control $u_{f}$;

- a sharing function $k$;

- a safe set $\mathcal{R}_{s} \triangleq \mathcal{P}_{a} \times \mathcal{A}_{s} \subset \mathcal{P}_{a} \times \mathcal{A} \triangleq \mathcal{R}$;

where $\mathcal{A}$ and $\mathcal{A}_{s}$ are the set of all heading angles and the set of all heading angles with which the robot is unable to reach the boundary of $\mathcal{P}_{a}$ within some arbitrary small time respectively, such that the s-closed-loop system has the following properties.

P1) The set $\mathcal{R}$ is forward invariant.

P2)

$$
\Omega_{s}= \begin{cases}\Omega_{h} & \text { if } \Omega_{h} \subset \mathcal{R}_{s}, \\ \Pi_{\mathcal{R}_{s}}\left(\Omega_{h}\right) & \text { if } \Omega_{h} \not \subset \mathcal{R}_{s},\end{cases}
$$

where $\Pi_{\mathcal{R}_{s}}\left(\Omega_{h}\right)$ is the projection of $\Omega_{h}$ into the set $\mathcal{R}_{s}$, which will be defined in Section III-A.

P3) $u_{s}=u_{h}$ if the state of the s-closed-loop system is in $\mathcal{R}_{s}$.

Note that the problem statement for the dynamic model (2) is similar to the above one except for that the safe set $\mathcal{R}_{s}$ is defined as $\mathcal{R}_{s} \triangleq \mathcal{P}_{a} \times \mathcal{A}_{s} \times \mathcal{V}_{s} \times \mathcal{W} \subset \mathcal{P}_{a} \times \mathcal{A} \times \mathcal{V} \times \mathcal{W}_{j} \triangleq \mathcal{R}$ and $\Pi_{\mathcal{R}_{s}}\left(\Omega_{h}\right)$ will be defined in Section IV-A. $\mathcal{V}$ and $\mathcal{W}$ are the sets of linear velocities and angular velocities, respectively. In addition, for any fixed $\theta$ and any $(x, y) \in \mathcal{P}_{a}, \mathcal{V}_{s}$ and $\mathcal{W}_{s}$ are the sets of all linear velocities and angular velocities with which the robot is unable to reach the boundary of $\mathcal{P}_{a}$ within some arbitrary small time, respectively.

\section{SHARED-CONTROL FOR THE KINEMATIC MODEL OF A MOBILE ROBOT}

This section gives a solution to the shared-control problem for the kinematic model of a mobile robot described by (1) with any non-empty $\mathcal{P}_{a}$.

\section{A. Design of the f-control}

Since measurements of the absolute positions are not available and we can only measure the distances to obstacles and the angular differences between the actual and the reference heading angles, the dynamics of the system (1) can be rewritten using the variables $d=\left[d_{1}, d_{2}\right]^{T}$ and $\theta_{e}$ defined in Section II. If $d_{2}=d_{2 l}$, then the system controlled by the feedback

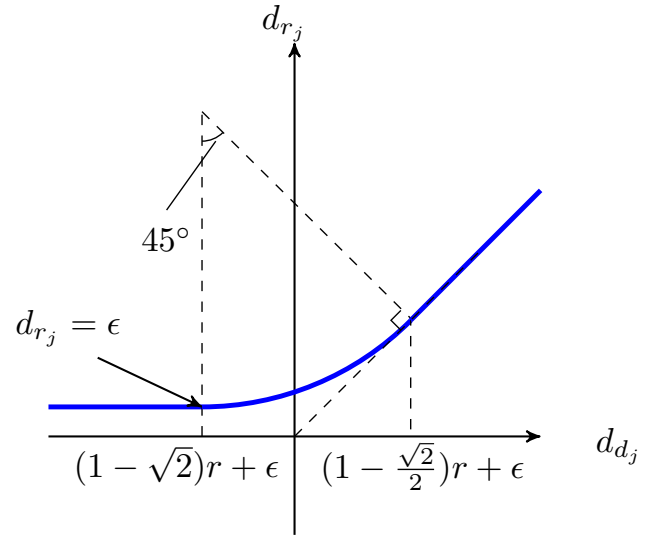

Fig. 4. The graph of the function $d_{r_{j}}$ given by (8).

controller can be described by the equations

$$
\begin{aligned}
& \dot{d}_{1}=-v_{f} \cos \theta_{e}, \\
& \dot{d}_{2}=-v_{f} \sin \theta_{e}, \\
& \dot{\theta}_{e}=\omega_{f} .
\end{aligned}
$$

Otherwise, if $d_{2}=d_{2 r}$, then the system controlled with the feedback controller can be described by the equations

$$
\begin{aligned}
& \dot{d}_{1}=-v_{f} \cos \theta_{e} \\
& \dot{d}_{2}=v_{f} \sin \theta_{e}, \\
& \dot{\theta}_{e}=\omega_{f} .
\end{aligned}
$$

Without loss of generality, we only discuss how to design the feedback controller in the case $d_{2}=d_{2 l}$. Since we regard the mobile robot as a point, the position constraint $(x, y) \in \mathcal{P}_{a}$ can be rewritten as

$$
d_{j} \geq 0 \quad \forall j \in\{1,2\} .
$$

Define the variable $z=\left[z_{1}, z_{2}\right]^{T}$ as

$$
z_{j}=\log \frac{d_{j}}{d_{r_{j}}}
$$

for all $j \in\{1,2\}$, where $d_{r_{j}}$, describing the reference trajectory for the state $d_{j}$, is defined as

$$
d_{r_{j}}= \begin{cases}d_{d_{j}}, & \text { if } d_{d_{j}} \geq\left(1-\frac{\sqrt{2}}{2}\right) r+\epsilon, \\ \epsilon, & \text { if } d_{d_{j}} \leq(1-\sqrt{2}) r+\epsilon, \\ m_{j}, & \text { otherwise }\end{cases}
$$

for all $j \in\{1,2\}$, with $m_{j}=(r+\epsilon)-$ $\sqrt{r^{2}-\left[(\sqrt{2}-1) r-\epsilon+d_{d_{j}}\right]^{2}}$ and a positive constant $\epsilon$. Note that the variable $d_{r_{j}}$, instead of $d_{d_{j}}$, is used in the definition of $z_{j}$, since $d_{d_{j}}$ may be unfeasible, i.e. there may exist a $t>0$ such that $d_{d_{j}}(t)<0$.

Fig. 4 illustrates the definition of $d_{r_{j}}$, from which it is clear that $d_{r_{j}}$ is a smooth function with positive values. As a result, $\dot{d_{r_{j}}}$ exists and

$$
\left.\dot{d}_{r_{1}}=-v_{r}^{i}, \quad \theta_{r}=\operatorname{atg}\left(-d_{r_{2}},-d_{r_{1}}\right)\right), \quad \dot{\theta}_{r}=\omega_{r} .
$$


Note that the definition of the safe set $\mathcal{R}_{s}$ for the system states of the kinematic model of the mobile robot given in Section II are relative to the $(x, y)$ coordinates. However, it can be transfered into the $\left(d_{1}, d_{2}, \theta\right)$ coordinates as follows.

Suppose $\left(d_{d}, \theta_{d}\right)$ is a point of the $\Omega$-limit set of the h-closedloop system, i.e. $\left(d_{d}, \theta_{d}\right) \in \Omega_{h}$, and define the projection of $\left(d_{d}, \theta_{d}\right)$ into $\mathcal{R}_{s}$ as

$$
\Pi_{\mathcal{R}_{s}}\left(d_{d}, \theta_{d}\right)=\left(d_{r}, \theta_{r}\right),
$$

where $d_{r}=\left[d_{r_{1}}, d_{r_{2}}\right]^{T}$ and $\theta_{r}$ are defined by (8) and (9), respectively. Then the projection of $\Omega_{h}$ into the safe set $\mathcal{R}_{s}$ is defined by

$$
\Pi_{\mathcal{R}_{s}}\left(d_{d}, \theta_{d}\right)=\left\{s \in \mathcal{R}_{s} \mid s=\Pi_{\mathcal{R}_{s}}\left(d_{d}, \theta_{d}\right), \forall\left(d_{d}, \theta_{d}\right) \in \Omega_{h}\right\} .
$$

Let $\theta_{e}^{*}=\operatorname{atg}\left(\gamma_{2} d_{2} z_{2}, d_{1}\left(\frac{v_{r}}{d_{r_{1}}}+\gamma_{1} z_{1}\right)\right)$, where $\gamma_{1}>0$ and $\gamma_{2}>0$. Note that $\theta_{e}^{*}(t)$ is calculated from the back-stepping method and it describes the desired value for $\theta_{e}$ at the time instant $t$. Consider the Lyapunov function relative to $d_{2}=d_{2 l}$ $L\left(z_{1}, z_{2}, \theta_{e}\right)$ given as

$$
L\left(z_{1}, z_{2}, \theta_{e}\right)=\frac{1}{2}\left[z_{1}^{2}+z_{2}^{2}+\left(\theta_{e}-\theta_{e}^{*}\right)^{2}\right],
$$

and choose $v_{f}$ and $\omega_{f}$ such that $\dot{L}<0$ for all $\left(z_{1}, z_{2}\right) \neq(0,0)$ and $\theta_{e} \neq \theta_{e}^{*}$. One such a choice is given by

$$
\begin{aligned}
v_{f}= & \sqrt{d_{1}{ }^{2}\left(\frac{v_{r}}{d_{r_{1}}}+\gamma_{1} z_{1}\right)^{2}+\gamma_{2}^{2} d_{2}{ }^{2} z_{2}{ }^{2}}, \\
\omega_{f}= & \dot{\theta}_{e}^{*}-\gamma_{3}\left(\theta_{e}-\theta_{e}^{*}\right)-\frac{z_{1} v_{f} \sin \frac{\theta_{e}+\theta_{e}^{*}}{2} \operatorname{sinc} \frac{\theta_{e}-\theta_{e}^{*}}{2}}{d_{1}} \\
& +\omega_{r}+\frac{z_{2} v_{f} \cos \frac{\theta_{e}+\theta_{e}^{*}}{2} \operatorname{sinc} \frac{\theta_{e}-\theta_{e}^{*}}{2}}{d_{2}},
\end{aligned}
$$

yielding

$$
\dot{L}=-\gamma_{1} z_{1}^{2}-\gamma_{2} z_{2}^{2}-\gamma_{3}\left(\theta_{e}-\theta_{e}^{*}\right)^{2} \leq 0 .
$$

This can be pushed back to the $\left(d_{1}, d_{2}, \theta_{e}\right)$ coordinates yielding

$$
\begin{aligned}
v_{f}= & \sqrt{d_{1}^{2}\left(\frac{v_{r}}{d_{r_{1}}}+\gamma_{1} \log \frac{d_{1}}{d_{r_{1}}}\right)^{2}+\gamma_{2}^{2} d_{2}^{2}\left(\log \frac{d_{2}}{d_{r_{2}}}\right)^{2},} \\
\omega_{f}= & -\frac{\log \frac{d_{1}}{d_{r_{1}}} v_{f} \sin \frac{\theta_{e}+\theta_{e}^{*}}{2} \operatorname{sinc} \frac{\theta_{e}-\theta_{e}^{*}}{2}}{d_{1}} \\
& +\frac{\log \frac{d_{2}}{d_{r_{2}}} v_{f} \cos \frac{\theta_{e}+\theta_{e}^{*}}{2} \operatorname{sinc} \frac{\theta_{e}-\theta_{e}^{*}}{2}}{d_{2}} \\
& +\dot{\theta}_{e}^{*}-\gamma_{3}\left(\theta_{e}-\theta_{e}^{*}\right)+\omega_{r},
\end{aligned}
$$

where

$$
\theta_{e}^{*}=\operatorname{atg}\left(\gamma_{2} d_{2} \log \frac{d_{2}}{d_{r_{2}}}, d_{1}\left(\frac{v_{r}}{d_{r_{1}}}+\gamma_{1} \log \frac{d_{1}}{d_{r_{1}}}\right) .\right.
$$

We are now ready to present a preliminary result.

Lemma 1: Consider the f-closed-loop system (1) with $\left[v_{s}, \omega_{s}\right]^{T}=\left[v_{f}, \omega_{f}\right]^{T}, d_{r}$ and $\left(v_{r}, \omega_{r}\right)$ given by (11), (8) and (9), respectively. Assume $(x(0), y(0)) \in \mathcal{P}_{a}$. Then the closedloop system has the following properties.

- $d_{1}(t)>0, d_{2}(t)>0$ for all $t \geq 0$;

- $\lim _{t \rightarrow \infty}\left(d_{1}(t)-d_{r_{1}}(t)\right)=\lim _{t \rightarrow \infty}\left(d_{2}(t)-d_{r_{2}}(t)\right)=0$.

Proof: According to the definition of $z_{j}$ given in (7), $d_{j}(t)>0$ for all $t \geq 0$ and $j \in\{1,2\}$. The first claim thus holds.

Consider the Lyapunov function (10) and note that $\dot{L}(t)<0$ for all $z \neq 0$ and $\theta_{e} \neq 0$. As a result $z$ and $\theta_{e}$ asymptotically converge to 0 and $\theta_{r}$, respectively, which proves the second property.

\section{B. Shared Control}

For any given human input $v_{h}$, the safe, hysteresis and dangerous subsets, $\mathcal{R}_{s}, \mathcal{R}_{h}$ and $\mathcal{R}_{d}$, are defined in equations (12) at the top of the next page where $D$ is the distance to the obstacle along the direction of $v_{h}$ (see Figure 1) and $b_{2}>b_{1}>0$ are user selected parameters. The three subsets have the following properties.

- For any fixed $v_{h}$, the union of the safe, the hysteresis and the dangerous set coincides with the overall feasible state space, i.e. $\mathcal{R}_{s}\left(v_{h}\right) \cup \mathcal{R}_{h}\left(v_{h}\right) \cup \mathcal{R}_{d}\left(v_{h}\right)=\mathcal{R}\left(v_{h}\right)$.

- For any fixed $v_{h}$, there is no intersections between the safe subset and the dangerous subset, i.e. $\mathcal{R}_{s}\left(v_{h}\right) \cap$ $\mathcal{R}_{d}\left(v_{h}\right)=\emptyset$.

For each group of constraints, the sharing function $k$ can be defined as (see [28])

$$
k\left(D, v_{h}\right)= \begin{cases}1, & \left(d_{1}, d_{2}, \theta_{e}\right) \in \mathcal{R}_{s}\left(v_{h}\right), \\ l, & \left(d_{1}, d_{2}, \theta_{e}\right) \in \mathcal{R}_{h}\left(v_{h}\right), \\ 0, & \left(d_{1}, d_{2}, \theta_{e}\right) \in \mathcal{R}_{d}\left(v_{h}\right),\end{cases}
$$

where

$$
l= \begin{cases}1, & \text { if }\left(d_{1}, d_{2}, \theta_{e}\right) \text { enters } \mathcal{R}_{h}\left(v_{h}\right) \text { from } \mathcal{R}_{s}\left(v_{h}\right), \\ 0, & \text { if }\left(d_{1}, d_{2}, \theta_{e}\right) \text { enters } \mathcal{R}_{h}\left(v_{h}\right) \text { from } \mathcal{R}_{d}\left(v_{h}\right) .\end{cases}
$$

Finally, the overall shared-control for the system (4) is given by

$$
u_{s}=\left(1-k\left(D, v_{h}\right)\right) u_{f}\left(d, d_{r}, \theta_{r}, \omega_{r}, v_{r}\right)+k\left(D, v_{h}\right) u_{h} .
$$

Theorem 1: Consider the kinematic model of a mobile robot (1) with the shared-control law given by (11)-(13)-(14). Assume $p(0)=[x(0), y(0)]^{T} \in \mathcal{P}_{a}$ and $u_{h}$ is a given $\mathrm{h}$ control. Then there exist $\gamma_{i}>0$, for all $i \in\{1,2\}$ and $b_{2}>b_{1}>0$ such that the s-closed-loop system has the following properties. 


$$
\begin{gathered}
\mathcal{R}_{s}\left(v_{h}\right)=\left\{\left(d_{1}, d_{2}, \theta_{e}+\theta_{r}\right) \in \mathbb{R}^{+} \times \mathbb{R}^{+} \times \mathbb{S}: v_{h} \leq \frac{1}{b_{2}-D}-\frac{1}{b_{2}} \text { if } D \leq b_{2}\right\} \\
\mathcal{R}_{h}\left(v_{h}\right)=\left\{\begin{array}{r}
\left(d_{1}, d_{2}, \theta_{e}+\theta_{r}\right) \in \mathbb{R}^{+} \times \mathbb{R}^{+} \times \mathbb{S}: \quad \frac{1}{b_{2}-D}-\frac{1}{b_{2}}<v_{h}<\frac{1}{b_{1}-D}-\frac{1}{b_{1}} \text { and } D \leq b_{1} \\
\text { or } v_{h}>\frac{1}{b_{2}-D}-\frac{1}{b_{2}} \text { and } b_{1} \leq D \leq b_{2}
\end{array}\right\} \\
\mathcal{R}_{d}\left(v_{h}\right)=\left\{\left(d_{1}, d_{2}, \theta_{e}+\theta_{r}\right) \in \mathbb{R}^{+} \times \mathbb{R}^{+} \times \mathbb{S}: v_{h} \geq \frac{1}{b_{1}-D}-\frac{1}{b_{1}} \text { and } 0 \leq D \leq b_{1}\right\}
\end{gathered}
$$

i) $p(t)$ stays in $\mathcal{P}_{a}$ for all $t \geq 0$.

ii) $\Omega_{s}=\Pi_{\mathcal{R}_{s}}\left(\Omega_{h}\right)$.

iii) $u_{s}(t)=u_{h}(t)$ if the system state belongs to the safe subset at time $t$.

iv) The feedback-control input $u_{f}$ is bounded.

Proof: To begin with, as detailed in Section III-A, the f-control $u_{f}$ is such that the configuration of the system stays in the admissible region $\mathcal{P}_{a}$. Consider the Lyapunov function candidate $L$ given by (10). Due to the definition of $d_{2}$ given in (3) the switch from $d_{2}=d_{2 l}$ to $d_{2}=d_{2 r}$ (or vise versa) does not cause any discontinuity in the value of it. If $u_{s}$ does not switch from one feedback controller to the other, i.e. if $\left(d_{1}, d_{2}, \theta\right)$ leaves the dangerous subset $\mathcal{R}_{d}$ at $t=t_{1}$ and enters it again at $t=t_{2}$, then due to the existence of the hysteresis subset, there exists a positive $\delta t$ such that $u_{s}(t)=u_{f}(t)$ for all $t \in\left[t_{1}, t_{1}+\delta t\right]$. Let $\Delta t$ be the smallest time period, in which one of the feedback controller is active. Note that $\Delta t>0$. Then it is always possible to find positive constants $\gamma_{1}, \gamma_{2}$ and $\gamma_{3}$ such that

$$
\int_{t_{2}}^{t_{2}+\Delta t} \dot{L} d t \leq L\left(t_{1}\right)-L\left(t_{2}\right)
$$

i.e. $L\left(t_{2}+\Delta t\right) \leq L\left(t_{1}\right)$. Let $u_{f}^{i_{1}}, u_{f}^{i_{2}}, \ldots, u_{f}^{i_{n}}$ be a series of active feedback controllers and let $u_{f}^{i_{j}}$ be active for the time interval $\left(t_{i_{j}}, T_{i_{j}}\right]$, where $i_{j} \in\left\{1,2, \ldots, i_{n}\right\}$ for all $j \in$ $\{1,2, \ldots, n\}$. Note that $t_{i_{j+1}} \geq T_{i_{j}}$ for all $j \in\{1,2, \ldots, n-$ $1\}$. Therefore $0 \leq L^{i_{n}}\left(T_{i_{n}}\right) \leq \cdots \leq L^{i_{2}}\left(T_{i_{2}}\right) \leq L^{i_{1}}\left(T_{i_{1}}\right)$. Define the overall Lyapunov function $L(t)$ as

$$
L(t)=L^{i_{j}}(t), \text { if } t \in\left(t_{i_{j}}, T_{i_{j}}\right] .
$$

From the above analysis and [29] $L(t)$ is a multiple Lyapunov function and this implies that the configuration of the system with the feedback controller stays in the admissible set for all $t \geq 0$. In addition, the definition of $\mathcal{R}_{d}$ indicates that any trajectory enters the dangerous subset $\mathcal{R}_{d}$ before leaving $\mathcal{R}$ where $u_{f}$ is active. Therefore, the set $\mathcal{R}$ is a forward invariant set and claim i) holds.

If $\Omega_{h} \subset \mathcal{R}_{s}$, then claim ii) is a consequence of the general results in [28], and of the fact that $\Omega_{h}$ is the $\Omega$-limit set of both the h-closed-loop and the f-closed-loop systems (by assumption, the former, and by equations (7)-(10), the latter). Otherwise, if $\Omega_{h} \not \subset \mathcal{R}_{s}$, Lemma 1 indicates that the $\Omega$-limit set of the f-closed-loop system is $\Pi_{\mathcal{R}_{s}}\left(\Omega_{h}\right)$. Furthermore, equation (12) indicates that the trajectory of the system enters $\mathcal{R}_{d}$ where the feedback controller is active, hence driving the states of the system back to $\mathcal{R}_{s}$, before leaving the admissible set $\mathcal{R}$. Therefore, property ii) holds.

Statement iii) is a direct consequence of the definition of $u_{s}$.

Finally, $u_{f}$ given by (11) is chosen such that $z$ and $\theta_{e}$ asymptotically converge to zero. Therefore, $z$ is bounded. In addition, $d_{r_{j}}(t)$ given by (8) for $j \in\{1,2\}$ is a function with all positive values. This leads to the result $d(t) \geq \epsilon$ for all $t \geq 0$ and some positive constant $\epsilon$. Hence, $u_{f}$ is bounded.

Note that the third property in Theorem 1 is essential if the shared-controller is applied to a training system, such as a training wheelchair. In addition, this property allows the human operator to do whatever he/she wants as long as his/her behaviour is safe. This is important, for example in the application to toy cars.

\section{SHARED-CONTROL FOR THE DYNAMIC MODEL OF A MOBILE ROBOT}

In this section we discuss how to design a shared-controller for the dynamic model of the mobile robot satisfying all the properties presented in Section II with any non-empty admissible configuration set.

\section{A. Design of the f-control}

Consistently with Section III-A, we design the feedback controller for the case $d_{2}=d_{2 l}$. Define variables $z$ and $d_{r}$ as in (7) and (8), respectively. Then system (2) with the variable $z$ and $\theta_{e}$ can be rewritten as

$$
\begin{aligned}
\dot{z}_{1} & =\frac{\dot{d}_{1}}{d_{1}}-\frac{\dot{d}_{r_{1}}}{d_{r_{1}}}=\frac{v_{r}}{d_{r_{1}}}-\frac{v \cos \theta_{e}}{d_{1}}, \\
\dot{z}_{2} & =\frac{\dot{d}_{2}}{d_{2}}-\frac{\dot{d}_{r_{2}}}{d_{r_{2}}}=-\frac{v \sin \theta_{e}}{d_{2}}, \\
\dot{\theta}_{e} & =\omega_{e}, \\
\dot{v} & =u_{1 f}, \\
\dot{\omega}_{e} & =u_{2 f}-u_{2 r},
\end{aligned}
$$


where $u_{r}=\left[u_{1 r}, u_{2 r}\right]^{T}$ is the reference input signal and it is calculated as

$$
u_{1 r}=\ddot{d}_{r_{1}}, \quad u_{2 r}=\ddot{\theta}_{r}
$$

Similarly to what stated in Section III-A, even though the definition of the safe set $\mathcal{R}_{s}$ for the states of the dynamic model of the mobile robot is given based on the $(x, y)$ coordinates, it can be mapped into the $\left(d_{1}, d_{2}, \theta, v, \omega\right)$ coordinates. Suppose $\left(d_{d}, \theta_{d}, v_{d}, \omega_{d}\right)$ is a point of the $\Omega$-limit set of the hclosed-loop system, i.e. $\left(d_{d}, \theta_{d}, v_{d}, \omega_{d}\right) \in \Omega_{h}$, and define the projection of $\left(d_{d}, \theta_{d}, v_{d}, \omega_{d}\right)$ into $\mathcal{R}_{s}$ as

$$
\Pi_{\mathcal{R}_{s}}\left(d_{d}, \theta_{d}, v_{d}, \omega_{d}\right)=\left(d_{r}, \theta_{r}, v_{r}, \omega_{r}\right),
$$

where $d_{r}$ and $\theta_{r}, v_{r}, \omega_{r}$ are defined as in (8) and (9), respectively. Then the projection of $\Omega_{h}$ into the safe set $\mathcal{R}_{s}$ is defined by

$\Pi_{\mathcal{R}_{s}}\left(d_{d}, \theta_{d}, v_{d}, \omega_{d}\right)=\left\{\begin{array}{l|l}s \in \mathcal{R}_{s} & \begin{array}{c}s=\Pi_{\mathcal{R}_{s}}\left(d_{d}, \theta_{d}, v_{d}, \omega_{d}\right), \\ \forall\left(d_{d}, \theta_{d}, v_{d}, \omega_{d}\right) \in \Omega_{h}\end{array}\end{array}\right\}$.

Let

$$
\begin{aligned}
\theta_{e}^{*}= & \operatorname{atg}\left(\gamma_{2} d_{2} z_{2}, d_{1}\left(\frac{v_{r}}{d_{r_{1}}}+\gamma_{1} z_{1}\right)\right), \\
v^{*}= & \sqrt{\left(d_{1}\left(\frac{v_{r}}{d_{r_{1}}}\right)+\gamma_{1} z_{1}\right)^{2}+\left(\gamma_{2} d_{2} z_{2}\right)^{2},} \\
\omega_{e}^{*}= & \dot{\theta}_{e}^{*}-\frac{z_{1} v^{*}}{d_{1}} \sin \frac{\theta_{e}+\theta_{e}^{*}}{2} \operatorname{sinc} \frac{\theta_{e}^{i}-\theta_{e}^{*}}{2} \\
& +\frac{z_{2}^{i} v^{*}}{d_{2}} \cos \frac{\theta_{e}+\theta_{e}^{*}}{2} \operatorname{sinc} \frac{\theta_{e}-\theta_{e}^{*}}{2},
\end{aligned}
$$

where $\gamma_{1}>0$ and $\gamma_{2}>0$. Consider the Lypunov function

$L\left(z_{1}, z_{2}, \theta_{e}, v, \omega_{e}\right)=\frac{1}{2}\left[\begin{array}{c}z_{1}^{2}+z_{2}^{2}+\left(\theta_{e}-\theta_{e}^{*}\right)^{2}+\left(v-v^{*}\right)^{2} \\ +\left(\omega_{e}-\omega_{e}^{*}\right)^{2}\end{array}\right]$,

and choose $u_{f}=\left[u_{1 f}, u_{2 f}\right]^{T}$ such that $\dot{L} \leq 0$ and $\dot{L} \equiv 0$ implies that $\left(z_{1}, z_{2}, \theta_{e}, v, \omega_{e}\right)=(0,0,0,0,0)$. One such a choice is given by

$$
\begin{aligned}
& u_{1 f}=\dot{v}^{*}+\frac{z_{1}}{d_{1}} \cos \theta_{e}^{i}+\frac{z_{2}}{d_{2}} \sin \theta_{e}-\gamma_{3}\left(v-v^{*}\right), \\
& u_{2 f}=u_{2 r}+\dot{\omega}_{e}^{*}-\theta_{e}+\theta_{e}^{*}-\gamma_{4}\left(\omega_{e}-\omega_{e}^{*}\right),
\end{aligned}
$$

yielding

$$
\dot{L}=-\gamma_{1} z_{1}{ }^{2}-\gamma_{2} z_{2}{ }^{2}-\gamma_{3}\left(v-v^{*}\right)^{2}-\gamma_{4}\left(\omega_{e}-\omega_{e}^{*}\right)^{2} .
$$

This can be pushed back into the $\left(d_{1}, d_{2}, \theta_{e}, v, \omega_{e}\right)$ coordinates yielding

$$
\begin{gathered}
u_{1 f}=\dot{v}^{*}+\frac{\log \frac{d_{1}}{d_{r_{1}}}}{d_{1}} \cos \theta_{e}+\frac{\log \frac{d_{2}}{d_{r_{2}}}}{d_{2}} \sin \theta_{e}-\gamma_{3}\left(v-v^{*}\right), \\
u_{2 f}=u_{2 r}+\dot{\omega}_{e}^{*}-\theta_{e}+\theta_{e}^{*}-\gamma_{4}\left(\omega_{e}-\omega_{e}^{*}\right),
\end{gathered}
$$

where

$$
\begin{aligned}
\theta_{e}^{*}= & \operatorname{atg}\left(\gamma_{2} d_{2} \log \frac{d_{2}}{d_{r_{2}}}, d_{1}\left(\frac{v_{r}}{d_{r_{1}}}+\gamma_{1} \log \frac{d_{1}}{d_{r_{1}}}\right),\right. \\
v^{*}= & \sqrt{\left(d_{1}\left(\frac{v_{r}}{d_{r_{1}}}\right)+\gamma_{1} \log \frac{d_{1}}{d_{r_{1}}}\right)^{2}+\left(\gamma_{2} d_{2} \log \frac{d_{2}}{d_{r_{2}}}\right)^{2},} \\
\omega_{e}^{*}= & \dot{\theta}_{e}^{*}-\frac{\log \frac{d_{1}}{d_{r_{1}}} v^{*}}{d_{1}} \sin \frac{\theta_{e}+\theta_{e}^{*}}{2} \operatorname{sinc} \frac{\theta_{e}-\theta_{e}^{*}}{2} \\
& +\frac{\log \frac{d_{2}}{d_{r_{2}}} v^{*}}{d_{2}} \cos \frac{\theta_{e}+\theta_{e}^{*}}{2} \operatorname{sinc} \frac{\theta_{e}-\theta_{e}^{*}}{2} .
\end{aligned}
$$

Lemma 2: Consider the f-closed-loop system (2) with $\left[v_{s}, \omega_{s}\right]^{T}=\left[u_{1 f}, u_{2 f}\right]^{T}, d_{r}$ and $\left(v_{r}, \omega_{r}\right)$ given by (17), (8) and (9), respectively. Assume $(x(0), y(0)) \in \mathcal{P}_{a}$. Then the closed-loop system has the following properties.

- $d_{1}(t)>0, d_{2}(t)>0$ for all $t \geq 0$;

- $\lim _{t \rightarrow \infty}\left(d_{1}(t)-d_{r_{1}}(t)\right)=\lim _{t \rightarrow \infty}\left(d_{2}(t)-d_{r_{2}}(t)\right)=0$.

\section{B. Shared Control}

The definitions of the three subsets, the safe subset $\mathcal{R}_{s}$, the hysteresis subset $\mathcal{R}_{h}$ and the dangerous subset $\mathcal{R}_{d}$, are similar to that given in (12) except for that these three subsets are sets in $\left(d_{1}, d_{2}, \theta_{e}+\theta_{r}, v, \omega_{e}+\omega_{r}\right) \in \mathbb{R}^{+} \times \mathbb{R}^{+} \times \mathbb{S} \times \mathbb{R}^{2}$. In addition, the sharing function $k_{d}$ can be defined as in (13). Therefore, the overall shared-control for the system (2) is given, similarly to (14), by

$$
\begin{aligned}
u_{s}= & \left(1-k_{d}\left(D, v_{h}\right)\right) u_{f}\left(d, d_{r}, \theta_{e}, v, \omega_{e}, \theta_{r}, \omega_{r}, v_{r}, u_{r}\right) \\
& +k_{d}\left(D, v_{h}\right) u_{h} .
\end{aligned}
$$

Theorem 2: Consider the dynamic model of the mobile robot (2) with the shared-control law given by (13)-(17)-(18). Assume $p(0)=[x(0), y(0)]^{T} \in \mathcal{P}_{a}$ and $u_{h}$ is a given h-control. Then there exist $\gamma_{i}>0$, for all $i \in\{1,2,3,4\}$ and $b_{2}>b_{1}>0$ such that the s-closed-loop system has Property i) to iv) in Theorem 1.

Proof: The proof is similar to that of Theorem 1, hence it is omitted.

Remark 1: According to the shared-control law (12)-(13)-(14) and (12)-(13)-(18) for the kinematic and the dynamic model of the mobile robot, the feedback controller is active only if the robot is "close" to an obstacle. Therefore, $d_{j}$ can be modified by $\hat{d}_{j}=\min \left(d_{j}, \mathcal{B}\right)$, for $j \in\{1,2\}$, where $\mathcal{B}$ is a positive constant selected by the user.

Remark 1 indicates that we are not interested in the cases in which $d_{j}>\mathcal{B}$. This is very useful in applications, especially in the cases in which the obstacle is exactly parallel or perpendicular to the required direction, i.e. $d_{1}$ or $d_{2}$ equals infinity. 


\section{CASE STUDIES}

In this section we discuss three case studies: trajectory tracking in a static environment and a dynamic environment, and freedriving. In the first case the human is asked to drive the robot along a given trajectory from the initial position to a final target position in a static rectangular room, while in the second case a person is walking across the room. Finally, in the third case no task has been given to the operator and he/she "plays freely" with the robot. By comparing the simulation results and the experimental ones for the first and the second case study, we demonstrate that the trajectory for the robot in the experiment is close to that simulated, indicating the usefulness of the simulation. We provide only experimental results for the third case study. Note that we have only used the kinematic controller in the experiment because the robots available in the lab are all controlled directly by velocities.

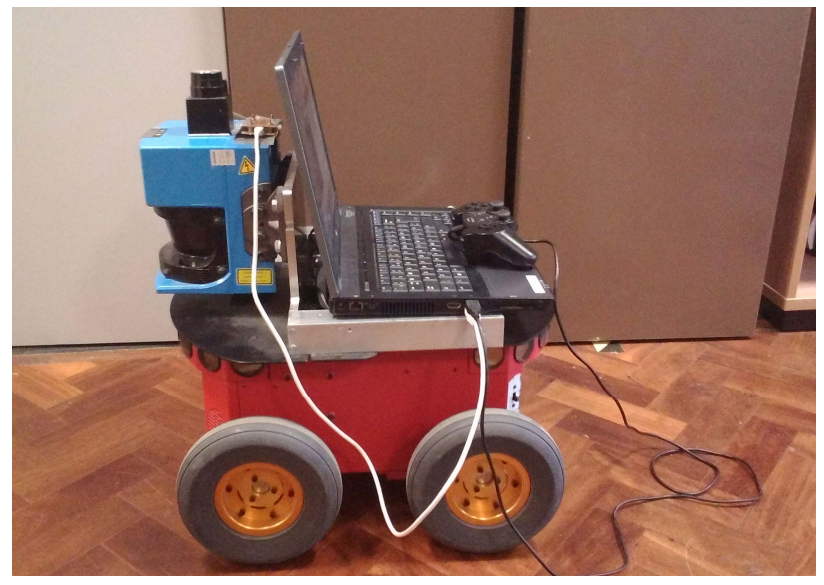

Fig. 5. The Experimental Setup

Both experiments are performed with a Pioneer 3-AT robot with the help of a robotic operative system named as Pioneer 2 Operative System (P2OS). We have used IMU to measure the angles, angular speed and magnetic field and lasers together with a Madgwick filter to measure the distances. 16 sonars (rate $=25 \mathrm{~Hz}$, detection range $\approx 20^{\circ}$ ) are used in the experiments, their positions are fixed facing outwards with 20 degree intervals. To estimate the positions, an extended Kalman filter has been used to combine the encoder data (which is inaccurate in long time) and the IMU data. In addition, the human input has been generated by the 'joystick' controller placed on the top of the computer in Figure 5. The overall system is illustrated in Fig. 5. The projection of the robot in the $(x, y)$-plane is a square with side length of $0.5 m \times 0.5 m$. We assume that the sensors are fixed to the middle of the two front-wheels and Figure 6 illustrates how the robot looks like in the $(x, y)$-plane.

The "Mobilerobots" platform uses a client-server mobile robot control architecture managed by the Pioneer 2 Operative System (P2OS) to insulate the developers from the lowest level of the motor control. In fact, it uses a PID controller with wheel encoder feedback to adjust a pulse width modulated (PWM) signal at the motor driver to control the power to the motors.

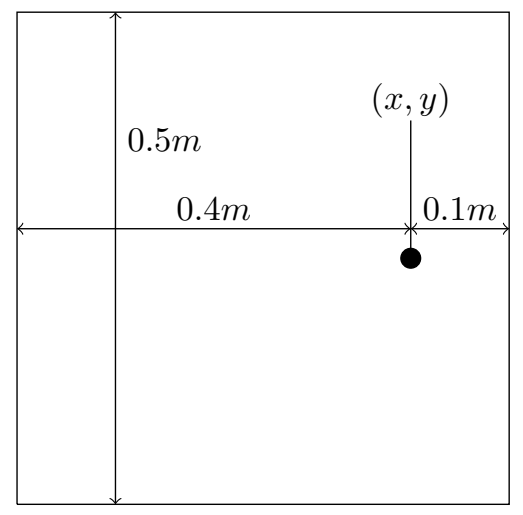

Fig. 6. Projection of the Robot in the $(x, y)$-Plane. Round mark: the sensor fixed on the robot. The right edge and the left edge of the square represent the front edge and the back edge of the robot, respectively.

It has been found that a fully loaded robot works best with $K_{p}=85, K_{v}=86, K_{i}=87$ and $K_{p}=82, K_{v}=83, K_{i}=$ 84 for the translation and rotation movement, respectively. To set up the experiment, we choose th sampling time to be $0.1 \mathrm{~s}$. The distance to the obstacle is calculated as $d=(c t) / 2$ where $c$ is the sound speed and $t$ is the time for receiving the echo.

\section{A. Case I: Trajectory Tracking}

Consider the kinematic model and the dynamic model of the mobile robot given by equation (1) and (2), respectively. Assume the admissible configuration set $\mathcal{P}_{a}$ is defined by

$\mathcal{P}_{a}=\left\{\begin{array}{l|l}(x, y) \mid \begin{array}{l}-0.2 \leq x \leq 2.9,-0.25 \leq y \leq 1.75 \\ y \in[-0.25,0.2] \cup[1.2,1.75] \text { if } x \in[0.7,2.4] \\ x \in[-0.2,0.7] \cup[2.4,2.9] \text { if } y \in[0.2,1.2]\end{array}\end{array}\right\}$,

and the reference trajectory is a straight line given by

$$
p_{d}(t)=\left[x_{d}(t), y_{d}(t)\right]^{T}=[0.067 t, 0.044 t]^{T} .
$$

This models the task in which the human user is restricted to drive the robot from its initial position $(0,0)$ to the final position $(3,2)$ along a straight line with a constant speed $0.08 \mathrm{~m} / \mathrm{s}$. Note that the desired trajectory is not always feasible, i.e. $\exists t>0$ such that $\left.p_{d}(t)\right) \notin \mathcal{P}_{a}$.

Simulation results are shown in Fig. 7 to Fig. 10. Fig. 7 demonstrates that the $(x, y)$ trajectory of the h-closed-loop system go through the non-admissible region (the grey shaded rectangle), while that of the s-closed-loop system move along the boundary of $\mathcal{P}_{a}$ until the reference trajectory becomes feasible. It also shows that the configuration of the robot without shared-control leaves $\mathcal{P}_{a}$ for a second time after $38 \mathrm{~s}$ as the red, dash-dotted and the yellow, solid, line indicate, while the robot with shared-control stops at the boundary of the admissible set. Fig. 8 and 9 show the time histories of the states and inputs of the h-closed-loop system and s-closedloop system. The functions $v(t)$ and $\omega(t)$ for the system (2) are smoother than those for the system (1) as they are system states rather than control inputs in the dynamic model of the mobile robot. Due to the discontinuity of $d_{r}$ caused by the 


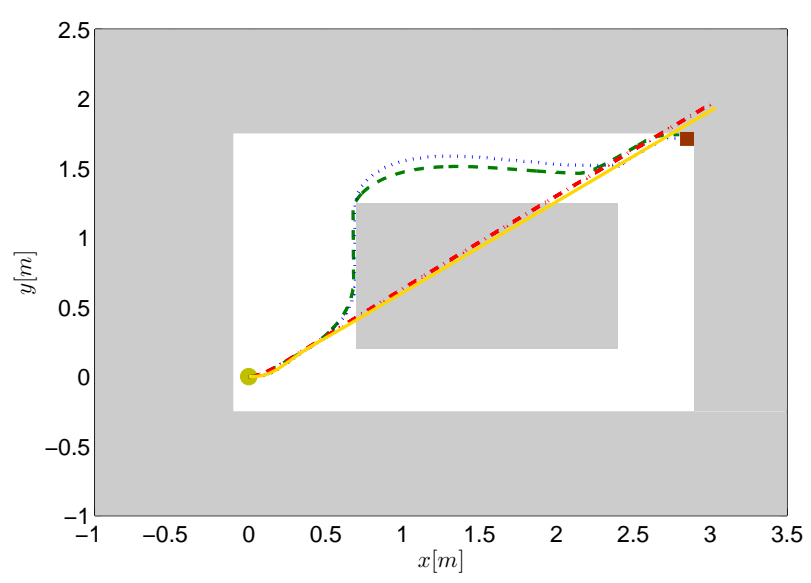

Fig. 7. $(x, y)$ trajectories of the system for the set $\mathcal{P}_{a}$ given by (19): h-closedloop (red, dash-dotted) and s-closed-loop (green, dashed) for the system (1), h-closed-loop (yellow, solid) and s-closed-loop (blue, dotted) for the system (2). Round mark: the initial position of the robot. Square mark: the final position of the robot with shared-control.
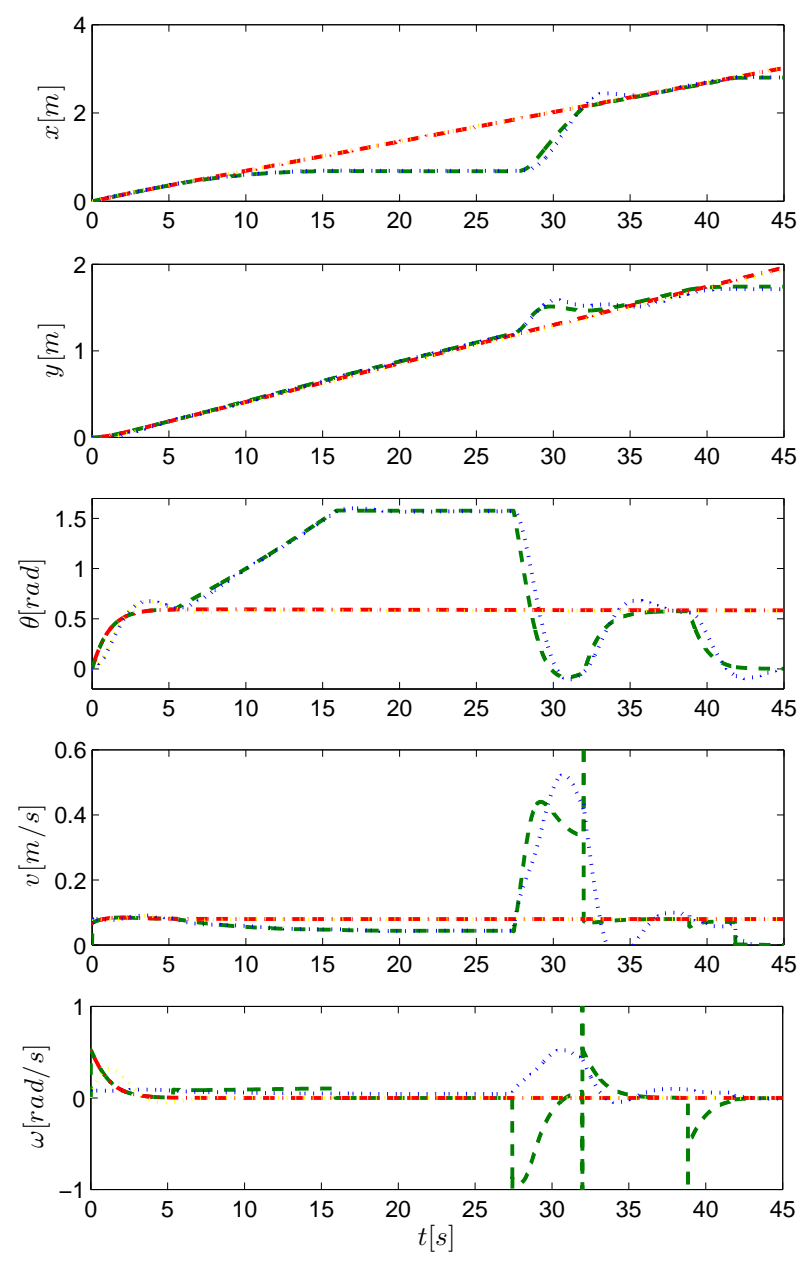

Fig. 8. Time histories of the variables $x, y, \theta, v$ and $\omega$ for the system with set $\mathcal{P}_{a}$ given by (19): h-closed-loop (red, dash-dotted) and s-closed-loop (green, dashed) for the system (1), h-closed-loop (yellow, solid) and s-closed-loop (blue, dotted) for the system (2).
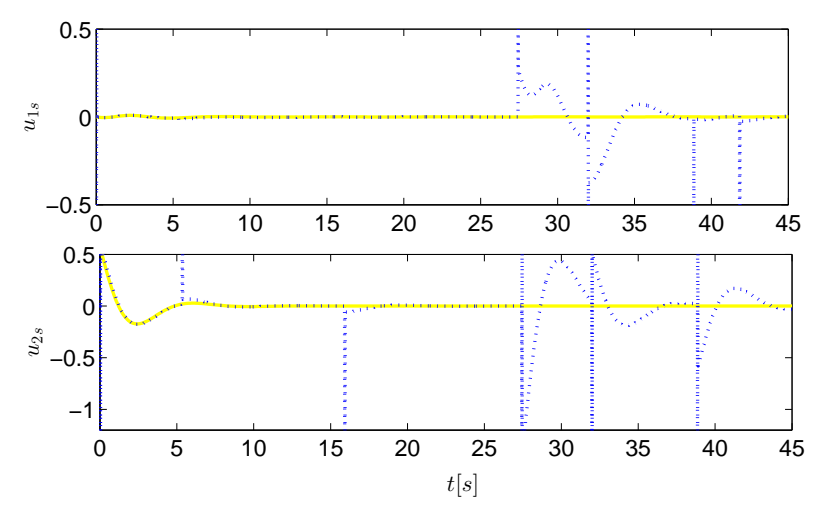

Fig. 9. Time histories of the inputs $u_{1}$ and $u_{2}$ for the system (2) with set $\mathcal{P}_{a}$ given by (19): h-closed-loop (yellow, solid) and s-closed-loop (blue, dotted).

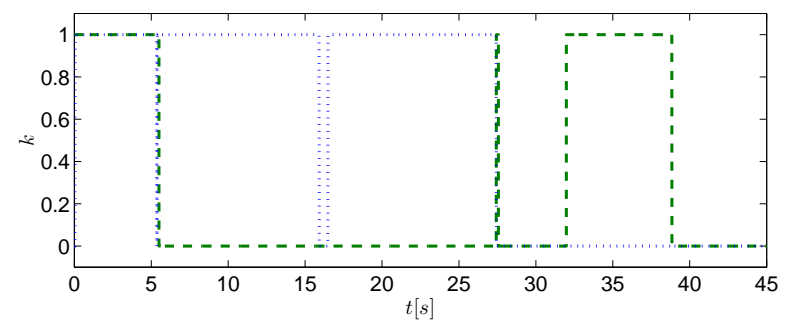

Fig. 10. Time histories of the sharing function $k$ for the system (1) (green, dashed) and (2) (blue, dotted) with the set $\mathcal{P}_{a}$ given by (19).

concavity of $\mathcal{P}_{a}$ and the fact that $v$ and $\omega$ change more gently for the dynamic model of the robot, the error between the configuration of the system (2) and its reference needs more time to converge to zero. Fig. 10 indicates that the feedback control is active for both system (1) and (2) at $5.5 s$ as $k$ changes its value to 0 . However, the control authority for the system (2) is passed to the human operator after a short period when the angular velocity of the robot is corrected by the feedback controller. In addition, due to the concavity of the feasible set $\mathcal{P}_{a}$, the robot needs to change its forward direction quickly to track the reference trajectory when $t \approx 16 \mathrm{~s}$. The feedback controller is active for less than 1 second to adjust the forward velocity and the angular velocity of the robot. Yet, the situation is different for the system (1). The control authority is hold by the feedback controller until the robot has left the obstacle, because the human has direct control on the velocities.

The experiment has been performed in a rectangular room with a table in it. Therefore, the admissible configuration region can be described as

$$
\mathcal{P}_{a}=\left\{\begin{array}{l|c}
(x, y) \mid \begin{array}{c}
-0.5 \leq x \leq 3,-0.5 \leq y \leq 2, \\
y \in[0,0.2] \cup[0.8,2] \text { if } x \in[1,2.5], \\
x \in[0,1] \cup[2.5,4.5] \text { if } y \in[0.2,0.8]
\end{array}
\end{array}\right\},
$$

where $(x, y)$ refers to every point of the robot. In other words, all the points of the robot should be located in the set $\mathcal{P}_{a}$ given by (21). Note that this set is equivalent to that described by (19) since $(x, y)$ in (19) refers to the center of the robot's front wheels. 
In the experiments, the linear velocity $v$ and the angular velocity $\omega$ of the car can be controlled directly by changing the left-wheel speed $v_{L}$ and the right-wheel speed $v_{R}$ as

$$
v=\frac{v_{R}+v_{L}}{2}, \quad \omega=\frac{v_{R}-v_{L}}{l},
$$

where $l$ is the length between the left-wheel and the rightwheel and equals to $0.5 \mathrm{~m}$ in the experiment. Therefore, the shared-control algorithm developed in Section III can be applied to control the robot.

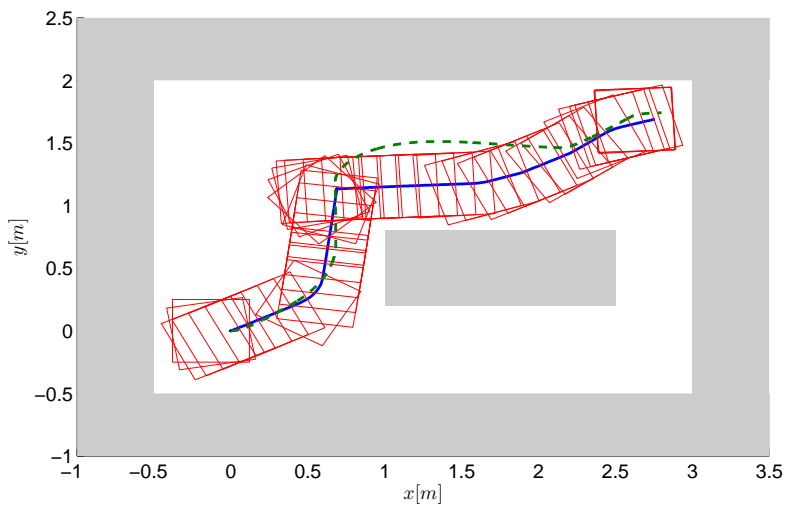

Fig. 11. The virtual path (green, dashed) generated in the simulation and the experimental path (blue, solid) of the mobile robot with the shared-control for the set $\mathcal{P}_{a}$ given by (21).
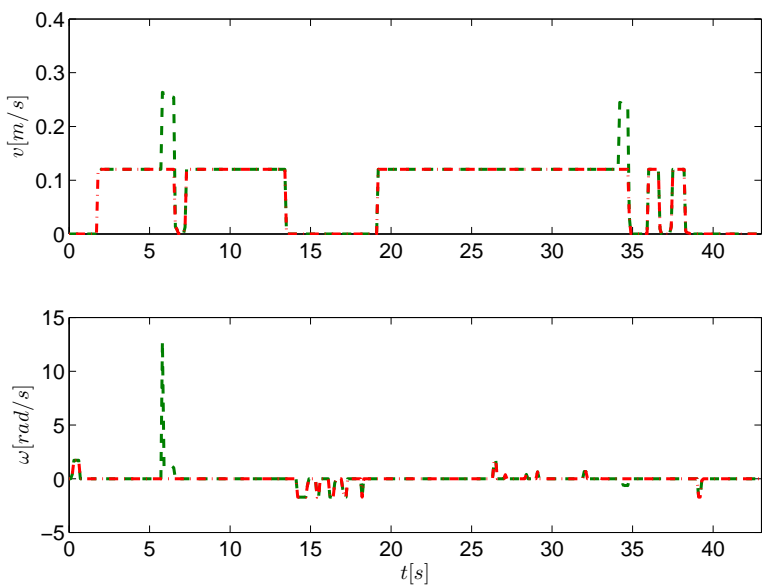

Fig. 12. Measured time histories of the inputs $v$ and $\omega$ for the h-closed-loop system (red, dash-dotted) and the s-closed-loop system (green, dashed).

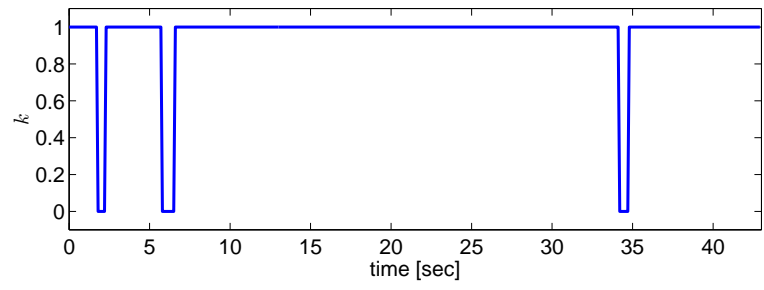

Fig. 13. Time history of the sharing function $k$ during the experiment.

Fig. 11 to 13 show the experimental results. Compared with the simulation results we note that there are slight differences

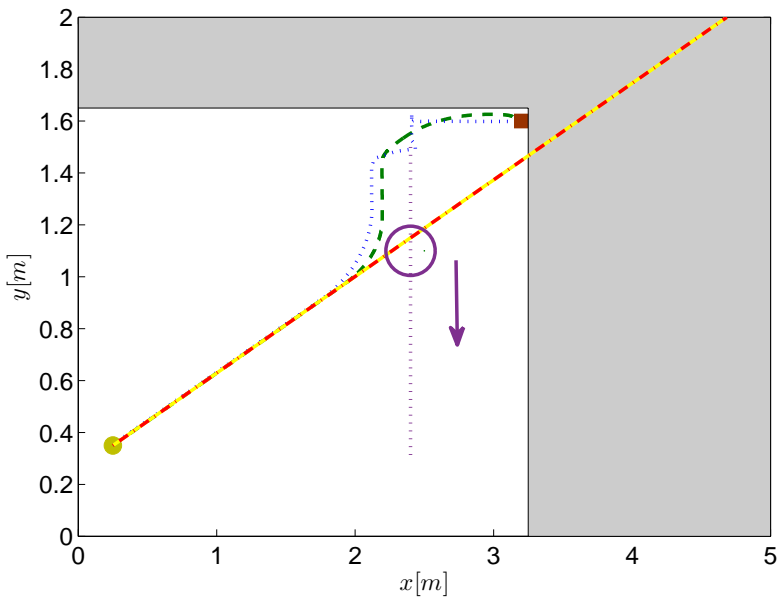

Fig. 14. $(x, y)$ trajectories of the system for the set $\mathcal{P}_{a}$ given by (22): h-closed-loop (red, dash-dotted) and s-closed-loop (green, dashed) for the system (1), h-closed-loop (yellow, solid) and s-closed-loop (blue, dotted) for the system (2). Round mark: the initial position of the robot. Square mark: the final position of the robot with shared-control. Purple circle: the moving obstacle. Purple, dotted curve: trajectory of the moving obstacle.

because in the simulation we assume that the $\mathrm{h}$-control is a controller making the robot moving towards the target point following a straight line and at a constant speed, while in the experiment this is done by the operator's intuition which is slightly different from the the h-control in the simulation. Fig. 13 shows that the time period when $u_{s}=u_{f}$ is shorter than the simulation results for the system (1) illustrated in Fig. 10. This is because in the experiment the human operator has noticed that he/she has to change the direction of the robot to avoid collisions after feedback from the signal $k$. In addition, the average velocity in the experiment (approximately $0.12 \mathrm{~m} / \mathrm{s}$ ) is larger than that in the simulation (approximately $0.08 \mathrm{~m} / \mathrm{s}$ ) due to frictions.

\section{B. Case II: Moving Obstacles}

The second case study deals with moving obstacles. Consider the kinematic model and the dynamic model of the mobile robot given by equations (1) and (2), respectively. Assume the robot is able to move within a rectangular room defined by

$$
\mathcal{P}_{a}=\{(x, y) \mid x \leq 3.25, y \leq 1.65\},
$$

and there is moving obstacle, the shape of which is a circle with radius of $0.2 \mathrm{~m}$, going along a straight line from $(2.4,1.5)$ to $(2.4,0.3)$ with forward speed $v=0.1 \mathrm{~m} / \mathrm{s}$. Suppose the reference trajectory is a straight line described by

$$
p_{d}(t)=\left[x_{d}(t), y_{d}(t)\right]^{T}=[0.25+0.43 t, 0.35+0.16 t]^{T} .
$$

This models the task in which the human is asked to drive the robot from the initial position $(0.25,0.35)$ to its final position $(4.7,2)$ along a straight line with the speed $v=$ $0.45 \mathrm{~m} / \mathrm{s}$. Note that the final target position is unfeasible, i.e. $(4.7,2) \notin \mathcal{P}_{a}$. 

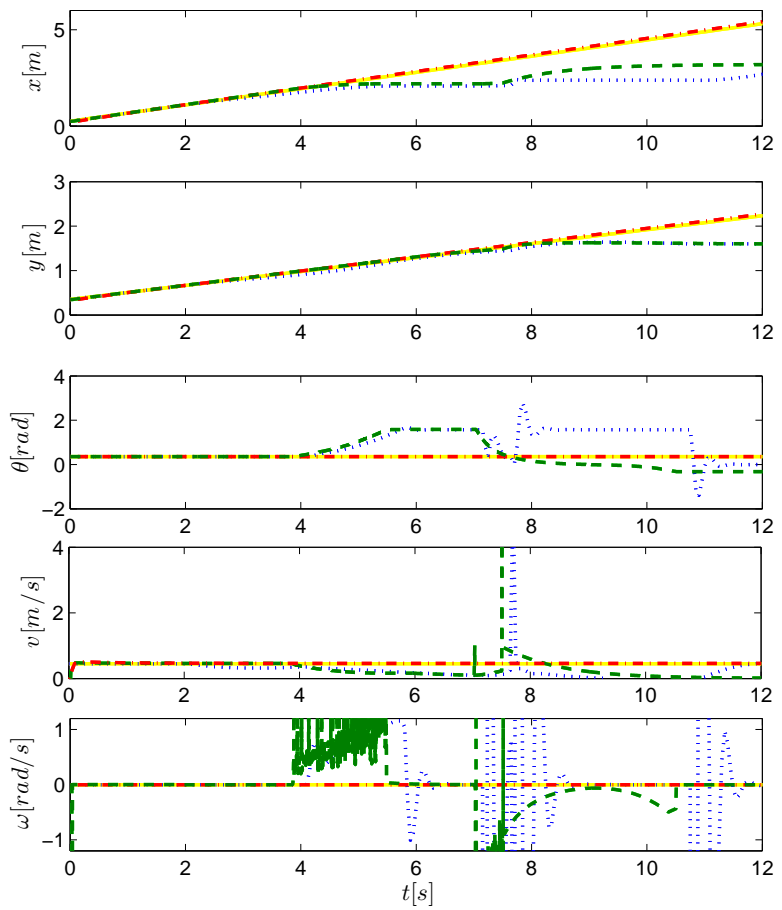

Fig. 15. Time histories of the variables $x, y, \theta, v$ and $\omega$ for the system with set $\mathcal{P}_{a}$ given by (22): h-closed-loop (red, dash-dotted) and s-closed-loop (green, dashed) for the system (1), h-closed-loop (yellow, solid) and s-closedloop (blue, dotted) for the system (2).
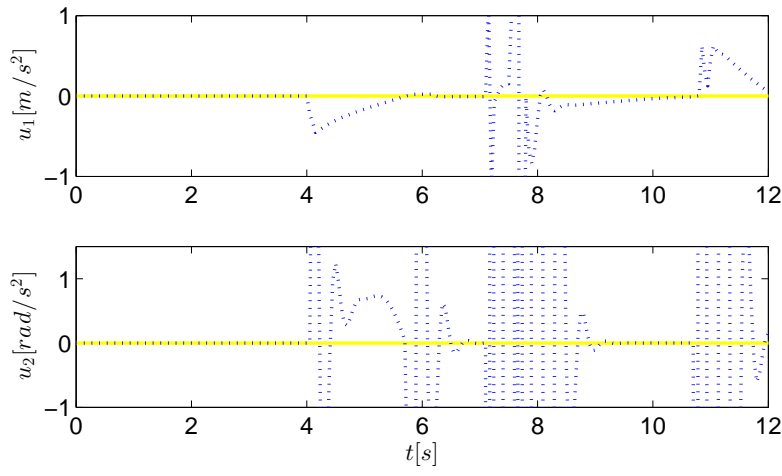

Fig. 16. Time histories of the inputs $u_{1}$ and $u_{2}$ for the system (2) with set $\mathcal{P}_{a}$ given by (22): h-closed-loop (yellow, solid) and s-closed-loop (blue, dotted).

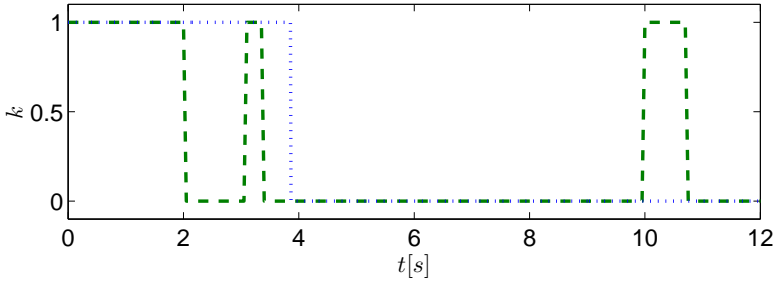

Fig. 17. Time history of the sharing function $k$ for the system (1) (green, dashed) and (2) (blue, dotted) with the set $\mathcal{P}_{a}$ given by (22).
Simulation results are displayed in Fig. 14 to Fig. 17. From Fig. 14 it is clear that the mobile robot with the shared-control changes its direction to avoid hitting the moving obstacle represented by the purple circle. Even though the purple circle is moving, Fig. 14 only shows its position when it starts to affect the robot (i.e. when it is close to the robot and force the feedback-controller to be active). Fig. 15 and Fig. 16 show the time histories of the states and inputs of the h-closedloop systems and the s-closed-loop systems. The blue time histories in Fig. 15 are smoother than the green time histories, especially for the function $v(t)$ and $\omega(t)$, because $v$ and $\omega$ are system states rather than input signals in system (2). In addition, the chattering in the control input $\omega$ illustrated in the bottom plot in Fig. 15 is mainly caused by the moving obstacle and can be reduced by predicting the trajectory of the moving obstacle. Due to the unfeasibility of the final position, the robot with the shared-control for both system (1) and (2) stops at the boundary of the rectangular room. When $t>11 \mathrm{~s}$, even if the human input $u_{h}$ is non-zero, the shared-control input of the system is $u_{s}=0$. Fig. 17 shows how the control authority is allocated between the human operator $(k=1)$ and the feedback controller $(k=0)$.

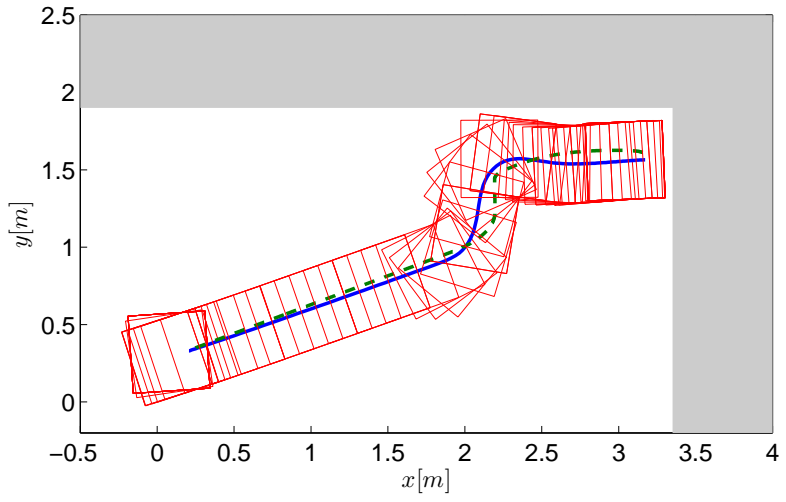

Fig. 18. The virtual path (green, dashed) and the experimental path (blue, solid) of the mobile robot with the shared-control for the set $\mathcal{P}_{a}$ given by (24)
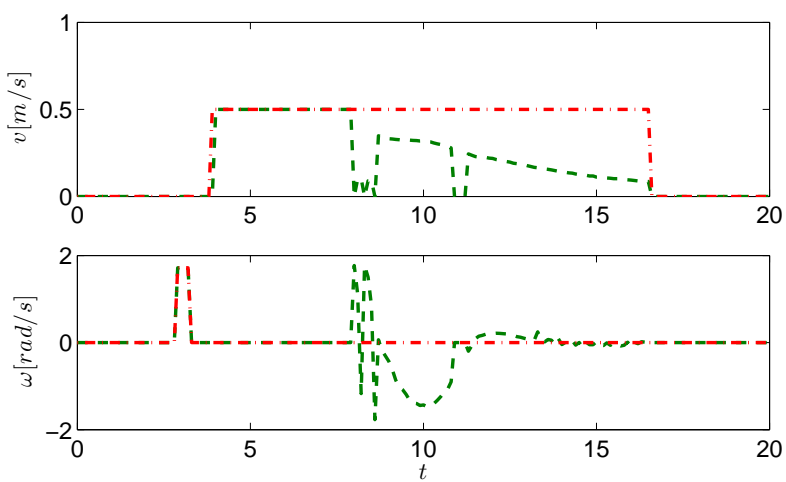

Fig. 19. Measured time histories of the inputs $v$ and $\omega$ for the h-closed-loop system (red, dash-dotted) and the s-closed-loop system (green, dashed).

The experiment has been done in a rectangular room described by

$$
\mathcal{P}_{a}=\{(x, y) \mid x \leq 3.35, y \leq 1.9\},
$$




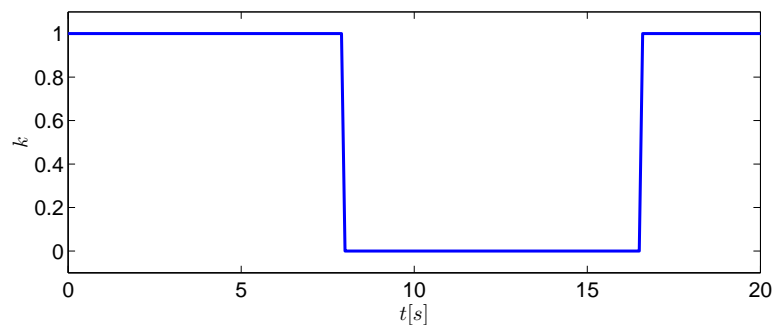

Fig. 20. Time history of the sharing function $k$ during the experiment.

where $(x, y)$ refers to every point of the robot. During the experiment a person is walking in the room with the speed of $0.1 \mathrm{~m} / \mathrm{s}$. Therefore, the experimental setting is the same as that given in the simulation part. Experimental results are demonstrated in Fig. 18 to Fig. 20. Fig. 18 shows that the robot with the shared-controller successfully avoid the collision with the pedestrian and walls, which meets our expectations and proves the effectiveness of the shared-control law. Fig. 19 shows that the shared-control input $v_{s}$ drops to 0 rather than decline gently according to its previous trend at $t=16 \mathrm{~s}$ because the human input $u_{h}$ falls to 0 suddenly. Fig. 20 illustrates the time period in which the feedback controller is active in the experiment.

\section{Case III: Free Driving}

The third case study is motivated by application to a toy car: the car is driven by the operator while away from "dangerous" situations. In real applications it is usually difficult to define the reference trajectory due to the unknown task and uncertainties of the environment. Therefore, we need to predict the desired trajectory based on the on-line measurement of the human inputs and the states.

In the experiment, the human operator has been asked to drive the robot freely without tracking any trajectory in a rectangular room with a table in it. The room can be described as

$\mathcal{P}_{a}=\left\{\begin{array}{l|l}(x, y) & \begin{array}{l}-0.5 \leq x \leq 4.4,-0.5 \leq y \leq 2 \\ y \in[-0.5,0.2] \cup[0.8,2] \text { if } x \in[1,2.5] \\ x \in[-0.5,1] \cup[2.5,4.4] \text { if } y \in[0.2,0.8]\end{array}\end{array}\right\}$.

Fig. 21 shows the $(x, y)$ trajectory of the robot with the sharedcontroller, while Fig. 22 illustrates how the inputs of the hclosed-loop system and the s-closed-loop system vary with time. In addition, Fig. 23 indicates how the control authority is shared between the human operator and the feedback controller. The experiment demonstrates the fact that the robot with the shared-control algorithm moves inside of the room and does not hit any obstacle (including both the table and the walls). Before any collision happens, the shared-controller would drive the robot moving along the obstacle until the states of the s-closed-loop system enter the safe region $\mathcal{R}_{s}$, where the human operator has the control authority. Fig. 22 shows that $v_{s}$ drops down to zero before $v_{h}$ becomes zero, which indicates that the robot stops in front of the wall even if the human input is non-zero when it is close to the wall and tends to hit the wall.

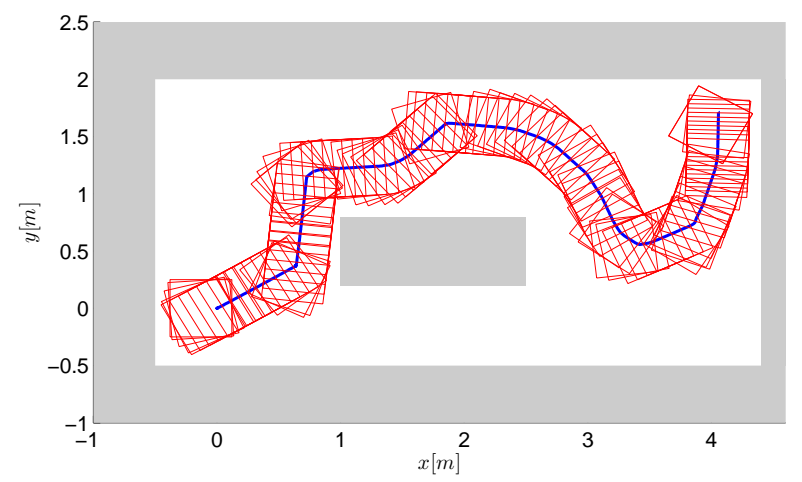

Fig. 21. Paths of the mobile robot with the shared-control for the set $\mathcal{P}_{a}$ (white area) given by (25).
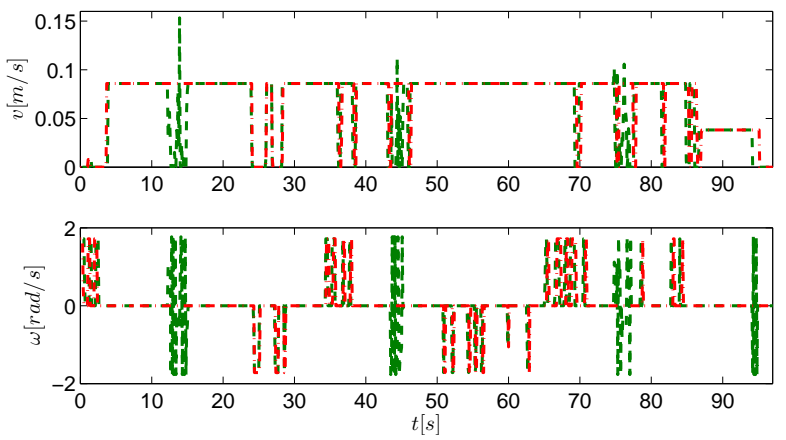

Fig. 22. Measured time histories of the inputs $v$ and $\omega$ for the h-closed-loop system (red, dash-dotted) and the s-closed-loop system (green, dashed).

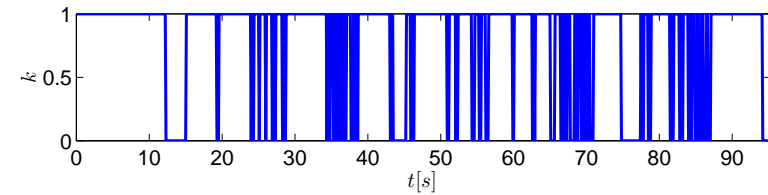

Fig. 23. Time history of the sharing function $k$ during the experiment.

\section{CONCLUSIONS}

We have developed a solution to the shared-control problem for the kinematic model and the dynamic model of a mobile robot without the knowledge of its absolute position. A hysteresis switch is built to integrate the human input and the feedback control input. The shared-control algorithm is developed for general admissible configuration sets with the assumption that $d_{d}$ is continuous. Both simulation results and experimental results with a Pioneer 3-AT robot show the effectiveness of the shared-control law. Future research will focus on the shared-control design for cars and multi-agent systems. 


\section{REFERENCES}

[1] S. Tzafestas, Introduction to Mobile Robot Control. Elsevier, 2014.

[2] T. Carlson and Y. Demiris, "Collaborative control for a robotic wheelchair: Evaluation of performance, attention, and workload," IEEE Transactions on Systems, Man and Cybernetics, Part B: Cybernetics, vol. 42, no. 3, pp. 879-888, 2012.

[3] T. Rofer and A. Lankenau, "Ensuring safe obstacle avoidance in a shared-control system," in Proc. of IEEE International Conference on Emerging Technologies and Factory Automation, vol. 1, Barcelona, 1999 , pp. 1405-1414.

[4] J. Kim, J. Lee, and Y. Choi, "LAROB: Laser-Guided Underwater Mobile Robot for Reactor Vessel Inspection," IEEE Trans. on Mechatronics, vol. 19, no. 4, pp. 1216-1225, 2013.

[5] H. Rezaee and F. Abdollahi, "A decentralized cooperative control scheme with obstacle avoidance for a team of mobile robots," IEEE Trans. on Industrial Electronics, vol. 61, no. 1, pp. 347-354, 2013.

[6] M. Seder, K. Macek, and I. Petrovic, "An integrated approach to realtime mobile robot control in partially known indoor environments," in Proc. of Annual Conference of IEEE Industrial Electronics Society, 2005, pp. $1785-1790$.

[7] K. Kanjanawanishkul and A. Zell, "Path following for an omnidirectional mobile robot based on model predictive control," in Proc. of IEEE International Conference on Robotics and Automation, 2009, pp. 33413346.

[8] A. Tahirovic and G. Magnani, "General Framework for Mobile Robot Navigation Using Passivity-Based MPC," IEEE Trans. on Automatic Control, vol. 68, no. 1, pp. 184-190, 2010.

[9] G. dos Reis, A. Siqueira, and M. Terra, "Nonlinear $H_{\infty}$ Control Via Quasi-LPV Representation and Game Theory for Wheeled Mobile Robots," in Proc. of IEEE International Symposium on Intelligent Control, Limassol, 2005, pp. 686-691.

[10] J. Xu, Z. Guo, and T. Lee, "Design and implementation of integral sliding-mode control on an underactuated two-wheeled mobile robot," IEEE Trans. on Industrial Electronics, vol. 61, no. 7, pp. 3671-3681, 2013.

[11] S. Yang, A. Zhu, G. Yuan, and M. Meng, "A bioinspired neurodynamicsbased approach to tracking control of mobile robots," IEEE Trans. on Industrial Electronics, vol. 59, no. 8, pp. 3211-3220, 2011.

[12] Z. Hou, A. Zou, L. Cheng, and M. Tan, "Adaptive control of an electrically driven nonholonomic mobile robot via backstepping and fuzzy approach," IEEE Trans. on Control Systems Technology, vol. 17, no. 4, pp. 803-815, 2009.

[13] R. Wai and Y. Lin, "Adaptive moving-target tracking control of a visionbased mobile robot via a dynamic petri recurrent fuzzy neural network," IEEE Trans. on Fuzzy Systems, vol. 21, no. 4, pp. 688-701, 2012.

[14] L. Leottau and M. Melgarejo, "A simple approach for designing a type2 fuzzy controller for a mobile robot application," in Proc. of Annual Meeting of the North American Fuzzy Information Processing Society, 2010, pp. 1-6.

[15] C. Juang and C. Hsu, "Reinforcement ant optimized fuzzy controller for mobile-robot wall-following control," IEEE Trans. on Industrial Electronics, vol. 56, no. 10, pp. 3931-3940, 2009.

[16] C. Samson, "Control of chained systems application to path following and time-varying point-stabilization of mobile robots," IEEE Trans. on Automatic Control, vol. 40, no. 1, pp. 64-77, 1995.

[17] Z. Qu, J. Wang, C. Plaisted, and R. Hull, "Global-stabilizing near- optimal control design for nonholonomic chained systems," IEEE Trans. on Automatic Control, vol. 51, no. 9, pp. 1440-1456, 2006

[18] H. Fujimoto, S. Yamakawa, and Y. Funahashi, "Switching control of chained systems based on time-state control form," in Proc. of IEEE Annual Conference on Industrial Electronics Society, vol. 2, 2000, pp. $1105-1110$.

[19] J. Jiang and A. Astolfi, "State and output-feedback shared-control for a class of linear constrained systems," Accepted by IEEE Trans. on Automatic Control.

[20] N. Uchiyama, T. Hashimoto, S. Sano, and S. Takagi, "Model-reference control approach to obstacle avoidance for a human-operated mobile robot," IEEE Trans. on Industrial Electronics, vol. 56, no. 10, pp. 3892 3896, 2009.

[21] L. Tonin, M. Tavella, S. Perdikis, and J. Millan, "The Role of SharedControl in BCI-Based Telepresence," in Proc. of IEEE Conference on Systems Man and Cybernetics, 2010, pp. 1462-1466.

[22] C. Masone, A. Franchi, H. Bulthoff, and P. Giordano, "Interactive planning of persistent trajectories for human-assisted navigation of mobile robots," in Proc. of IEEE International Conference on Intelligent Robots and Systems, Vilamoura, 2012, pp. 2641-2648.

[23] H. Rifa, M. Hua, T. Hamel, and P. Morin, "Haptic-based bilateral teleoperation of underactuated unmanned aerial vehicles," in Proc. of 18th IFAC World Congress, Milano, Italy, 2011, pp. 13782-13788.

[24] C. Masone, A. Franchi, H. H. Blthoff, and P. R. Giordano, "Shared trajectory planning for human-in-the-loop navigation of mobile robots in cluttered environments," in In 5th International Workshop on HumanFriendly Robotics, 2012.

[25] C. Masone, P. R. Giordano, H. H. Blthoff, and A. Franchi, "Semiautonomous trajectory generation for mobile robots with integral haptic shared control," in Proc. of IEEE International Conference on Robotics and Automation, Hong Kong, 2014, pp. 6468-6475.

[26] P. Stegagno, M. Basile, H. H. Blthoff, and A. Franchi, "A semiautonomous uav platform for indoor remote operation with visual and haptic feedback," in Proc. of IEEE International Conference on Robotics and Automation, Hong Kong, 2014, pp. 3862-3869.

[27] J. Jiang and A. Astolfi, "Shared-control for the kinematic model of a mobile robot," in Proc. of IEEE Conference on Decision and Control, Los Angeles, USA, 2014, pp. 62-67.

[28] C. Prieur, "Uniting local and global controllers with robustness to vanishing noise," Math. Control Signals Systems, vol. 14, pp. 143-172, 2001.

[29] D. Liberzon, Switching in Systems and Control. Birkhauser, Boston, 2003. 